\title{
MODELING THE MODELER: AN EMPIRICAL STUDY ON HOW MODELERS LEARN TO CREATE SIMULATIONS
}

\author{
Hamdi Kavak \\ Department of Computational and Data Sciences \\ George Mason University \\ 4400 University Dr. Fairfax, VA, USA \\ hkavak@gmu.edu
}

\author{
Jose Padilla \\ Saikou Diallo \\ Anthony Barraco \\ Virginia Modeling Analysis and Simulation Center \\ Old Dominion University \\ 1030 University Blvd., Suffolk, VA, USA \\ jpadilla, sdiallo, abarraco@odu.com
}

\begin{abstract}
This paper presents our novel efforts on automatically capturing and analyzing user data from a discreteevent simulation environment. We collected action data such as adding/removing blocks and running a model that enable creating calculated data fields and examining their relations across expertise groups. We found that beginner-level users use more blocks/edges and make more build errors compared to intermediate-level users. When examining the users with higher expertise, we note differences related to time spent in the tool, which could be linked to user engagement. The model running failure of beginnerlevel users may suggest a trial and error approach to building a model rather than an established process. Our study opens a critical line of inquiry focused on user engagement instead of process establishment, which is the current focus in the community. In addition to these findings, we report other potential uses of such user action data and lessons learned.
\end{abstract}

Keywords: simulation learning, modeling the modeler, simulation user analysis, web-based simulation, user engagement.

\section{INTRODUCTION}

Simulations play a vital role in training learners to gain experience in their profession (Cant and Cooper 2010) and making challenging topics easy to comprehend (Wieman, Adams, and Perkins 2008). In both simulation-based learning cases, learners are exposed to existing simulation models that are operated by the learner with minor creative efforts to improve domain-specific knowledge. While acknowledging the educational value of such simulations, we argue that simulations are equally critical tools to provide learners with creative thinking skills in addition to exposing them to science, technology, engineering, and math concepts. Such skills can be gained by allowing the learner to develop simulation models of their interest (Padilla et al. 2016).

Educational efforts such as the Scalable Game Design curriculum (Repenning et al. 2015) aims to expose K-12 students to Computer Science concepts by letting them create games and simulations which are motivational to keep students in "flow" (Csikszentmihalyi, Abuhamdeh, and Nakamura 2014). Their findings suggest that developing simulations helped students to have high motivation across different racial 
backgrounds while there is no evidence of whether students learned the concepts or not. Such learning progression can be captured through pre/post surveys or analyzing data collected from software tools automatically. While surveys have been used to assess student progression, they require the active participation of the learner and the educator (i.e., survey design). We note that computer science educators have started to follow the automated data collection and analysis path, which does not require learner participation or survey design (Fields et al. 2016). The main goal becomes turning user signals into meaningful insights for education purposes.

When it comes to modeling and simulation $(M \& S)$ education, such data collection efforts are scarce. We argue that many popular simulation tools and libraries are developed for desktop computers with no builtin user data collection mechanism. In contrast, web-based simulation environments are becoming more popular and have the potential to address this challenge.

In this paper, we report our efforts on understanding the progression of simulation learners. Specifically, we have implemented a data-collection mechanism on our web-based discrete-event simulation development environment called CLOUDES (Padilla et al. 2014), which automatically captures user actions. We collected over 100,000 such actions (with user-provided consent) and turned them into the knowledge of learners' progress. Since such efforts are scarce to find in the M\&S literature, we report our initial findings and lessons learned to shed light on future development. In the remainder of this paper, we first present the simulation environment used in this research (section 2). Second, we report on how we collected and processed such data as well as report data properties (section 3). Third, we show preliminary analysis using this data, which yields intuitive but interesting results (section 4). Finally, we report other potential ways to use this data and share lessons learned (section 5.)

\section{CLOUDES: WEB-BASED SIMULATION DEVELOPMENT ENVIRONMENT}

The modeler data collected in this paper is captured from a web-based discrete-event simulation development environment called CLOUDES (Padilla et al. 2014), which is located at http://cloudes.me. CLOUDES is designed to support the visual development of models, making it a suitable tool not only for experts but also for non-experts alike. In CLOUDES, users can start creating a model from scratch or use existing public models as the base model. With built-in unique features like conceptual modeling and input data collection, CLOUDES provides unique opportunities to develop models using a web browser readily available on mobile devices or traditional computers. Currently, CLOUDES is available in seven languages and serving users from more than 70 countries. Our previous efforts with CLOUDES include designing games to teach discrete-event simulation (Padilla et al. 2016), storytelling (Padilla et al. 2017), integrating sound in simulations to improve user experience (Deuro et al. 2017), and conceptualization (Padilla, Diallo, and Garcia 2018). A screenshot of the model development environment of CLOUDES is illustrated in Figure 1. 


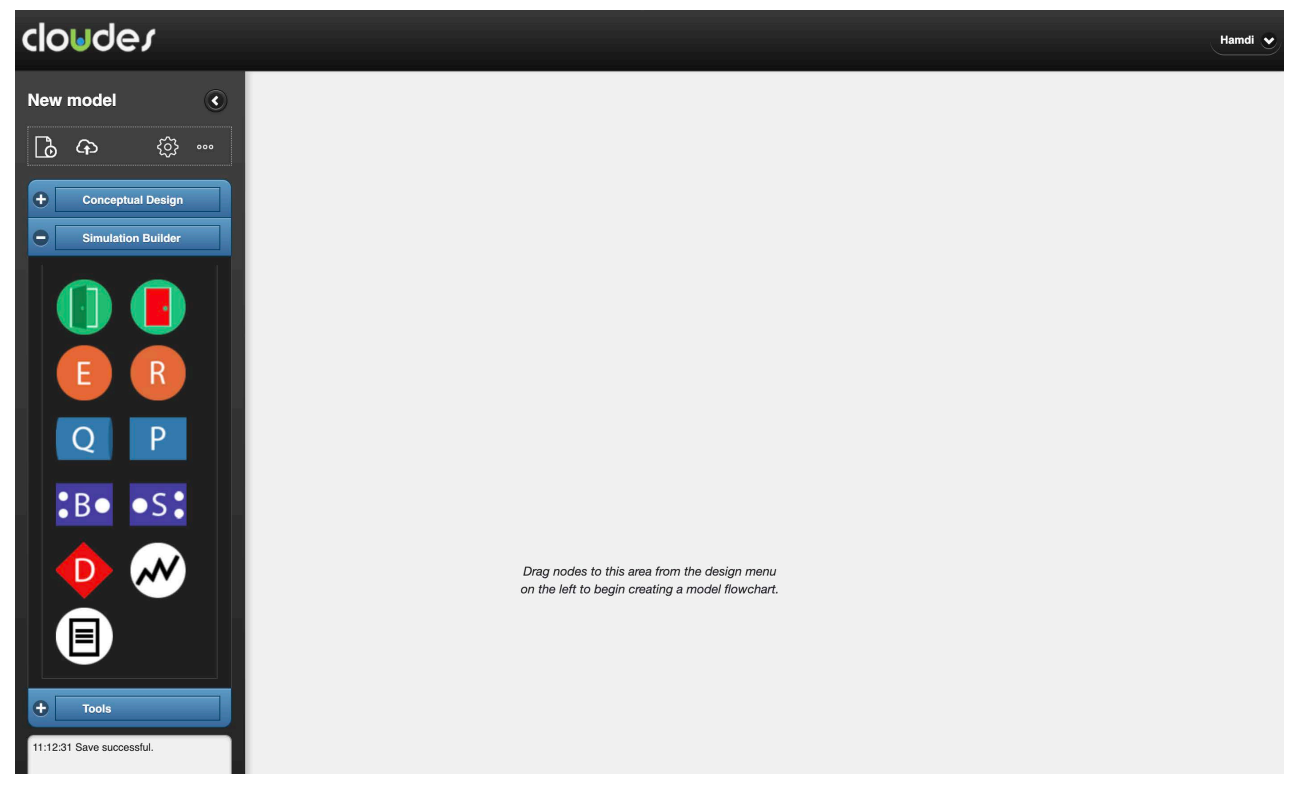

Figure 1: The web-based interface of CLOUDES.

There are eleven types of blocks in CLOUDES listed under the Simulation Builder tab. A user can dragand-drop them into the model development canvas. The first nine blocks are pertaining to model logic, while the last two are for visualization and description. A summary of these model blocks are as follows:

- Arrival: The block of which entities enter the system.

- Exit: Denotes entities leaving the system.

- Entity: The object that goes through the system (e.g., parts in an assembly line model).

- Resource: Represents things that use entities such as machines that assemble parts.

- Queue: This block is used when there is a chance to form a line of entities.

- Process: Denotes activities conducted on entities.

- Batch: Combines multiple entities.

- Separate: Breaks apart multiple combined entities.

- Decision: Allows splitting the path of entities.

- Chart: Visualization component for model states.

- Detailed Description: A textual description which can be placed on the canvas.

Once a block is added into the model development canvas, the user can double-click/tap to change its properties, such as the statistical distribution of inter-arrival times. Furthermore, certain block types can be wired to form a flow of entities within the model. In addition to adding nodes, CLOUDES allows users to run, save, and import models, share a model with other people, and display simulation results. Finally, a user can capture input data and create a schedule for the model. All these functionalities are available via the web user interface.

\section{USER DATA COLLECTION AND MANAGEMENT}

We have implemented a data collection mechanism in CLOUDES for the time period between June 2015 and December 2016. The overall data capture functionality is based on client-server communication. On the client-side, due to the nature of web-based systems, all user activities invoke some JavaScript events (e.g., button click). Thus, we listen to certain JavaScript events to capture actions of interest (e.g., adding/deleting a model block). Along with the action, we also acquire some additional parameters (e.g., model state), which may vary by action type. After parameters are prepared, we make a service call to the server-side and push the data. On the server-side, the incoming data is saved into a database. 
The details of the data and the way we store it follow common practices seen in web-based applications. We store collected data in a PostgreSQL database table where we call each record as action record and describe columns as below.

- Id column holds the unique identifier of the action record.

- ParticipantId and SessionId columns hold the user's encrypted identifier and session identifier, respectively.

- Time column holds the date and time when the action is performed by the user.

- Action column holds a number that represents one of the actions shown in Table 1.

- Data column holds additional text data in JSON format. We intentionally kept this column semistructured because the data to be stored here varies based on the action. For instance, when the action is build simulation, the data column keeps the latest state of the model and errors occurred during the build process while in $\log$ in action holds user browser version and time zone information.

- SimulationId column holds a number representing the identifier of the simulation loaded.

- PageId column holds a unique identifier for the page that user visits and this identifier changes when the user refreshes the current page or navigates to another page.

Table 1: User action types captured by the data collection mechanism.

\begin{tabular}{lllll}
\hline Log in & New simulation & Delete node & Open results & Share simulation \\
Log out & Save simulation & Session created & Close results & Convert conceptual model \\
Simulation loaded & Load simulation & Session destroyed & Export results & \\
Load profile & Import sim & Save counter & Run simulation & \\
Build simulation & Add node & Delete counter & Stop simulation & \\
\hline
\end{tabular}

\subsection{Dataset}

From users who give consent, we have collected their majority of simulation development actions with session and timestamp information. We have gathered a total of 102,394 event records and later reduced it to 76,126 after cleaning incomplete and unusable ones. These events are generated by 296 users whose expertise levels and areas of work are shown in Table 2.

Table 2: Breakdown of users by simulation expertise level (left) and area of work (right).

\begin{tabular}{|c|c|c|c|}
\hline Expertise level & Number of users & Area & Number of users \\
\hline NA & 2 & Educator & 45 \\
\hline Beginner & 213 & Student & 186 \\
\hline Intermediate & 57 & \multirow{2}{*}{$\begin{array}{l}\text { Simulation researcher and } \\
\text { practitioner }\end{array}$} & \multirow[t]{2}{*}{30} \\
\hline Advanced & 13 & & \\
\hline Expert & 11 & Other & 35 \\
\hline
\end{tabular}

The user breakdown shows that the majority of our user base consists of beginner-level simulation modelers followed by intermediate, advanced, and expert users. The most common area of users are students followed 
by educators and simulation researchers or practitioners. Since the expertise level is a crucial information for the analysis, we discarded two users with unknown expertise (NA). In the end, we had 70,801 event records.

We gathered a summary of simulation records describing 1,100 simulations developed by users mentioned above. We noticed that not all users contributed an equal number of simulations, so we plotted simulation per-user distribution in Figure 2. Many users in our dataset only have a few simulation summary records, while only a few users have over ten simulations. We fit our simulation per-user data to the Power-Law distribution and gathered the dashed line (exponent $=2.94$ with the lower cutoff value 6 ) as in Figure 2 . This distribution resembles user behavior in other technological platforms (Clauset, Shalizi, and Newman 2009).

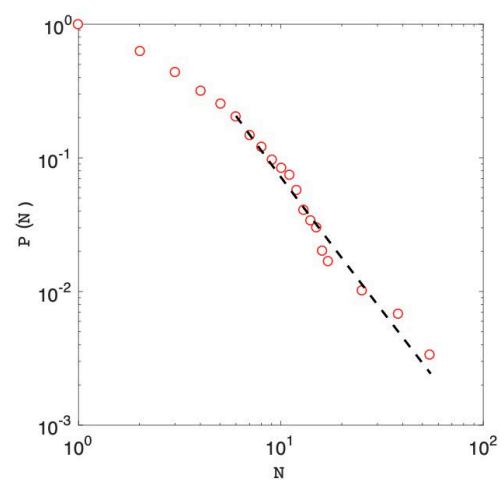

Figure 2: Simulation per user distribution.

\subsection{User Behavior Analysis Console}

In addition to the data collection mechanism, we have developed a user behavior analysis console. Our goal with designing the console is to make it easier to uncover the patterns of the process that users follow when they utilize CLOUDES. To achieve this goal, we implemented several functionalities in this console, including data process, preparation, selection, display, and export.

Data process and preparation functionality aims at transforming the raw data into a new form that can be used for visualization and analysis purposes. As the first step, we gather all participating users' lists from the database by querying distinct participant identifiers. As a result of this step, we gather users with properties, including expertise (e.g., beginner), industry (e.g., small business), and country. We then iterate through items of this participant list one by one in order to 1) get their entire raw action records and 2) generate a list of simulation activity records using raw action records. While gathering raw actions is a matter of performing a simple query, generating simulation activity records is a lengthy process.

In generating a simulation activity record, we first organize raw actions as lists by simulation identifiers. A simulation record may have multiple raw action lists. Second, each raw action list is then checked whether they include simulation creation or running activity. If not, we assume such actions as noise, which happens in cases when a user opens/creates a model and does not do any activity on it. If the raw action list, in fact, includes simulation creation or running activity, we generate a simulation activity record using the list of raw actions and add the record to the user. A simulation activity record contains a large number of parameters characterizing various aspects of the simulation, as shown in Table 3. 
Table 3: Simulation activity record parameters.

\begin{tabular}{|c|c|}
\hline & Parameters \\
\hline$\underset{\mathscr{D}}{\bar{D}}$ & $\begin{array}{l}\text { - User identifier (encrypted) } \\
\text { - User expertise } \\
\text { - User industry } \\
\text { - Model origin (self, template, public) } \\
\text { - Source simulation identifier } \\
\text { - Involves simulation creation and running }(\mathrm{C}, \mathrm{R}, \text { or } \mathrm{C}+\mathrm{R})\end{array}$ \\
\hline 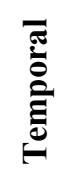 & $\begin{array}{l}\text { - Number of sessions } \\
\text { - Session lengths (longest, shortest, average, total) } \\
\text { - Number of days spent } \\
\text { - Time between two consecutive actions (longest, shortest, average) }\end{array}$ \\
\hline$\cdot \frac{\tilde{E}}{4}$ & $\begin{array}{l}\text { - Number of total actions } \\
\text { - Number of hits per button type (e.g., number of run button hits) } \\
\text { - Number of build error } \\
\text { - Number of additions/deletions per block type (e.g., number of queue block addition) } \\
\text { - Number of edge additions } \\
\text { - Number of session destroy action } \\
\text { - Number of page load action } \\
\text { - Number of logout action }\end{array}$ \\
\hline$\frac{n}{\varrho}$ & $\begin{array}{l}\text { - Number of inherited blocks per type (e.g., number of entity block inherited) } \\
\text { - Number of blocks per type (e.g., number of arrival) } \\
\text { - Number of blocks/edges converted from conceptual model }\end{array}$ \\
\hline 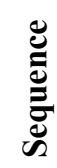 & $\begin{array}{l}\text { - Coded sequence of simulation creation actions (e.g., "ERQ-EP" represents Entity } \\
\text { added, Resource added, Queue added, Entity deleted, Process added) } \\
\text { - Code sequence of other actions (e.g., SS: Save Simulation; SD: Session Destroyed) } \\
\text { - Code sequence of all (simulation creation + other) actions }\end{array}$ \\
\hline
\end{tabular}

In order to serve the data to the analyst, the user behavior console provides a webpage with data preparation functionality described above. After data is prepared, we serve four main capabilities, including data 1) filter, 2) selection, 3) display, and 4) export.

The behavior analysis console provides several data filtering options. 1) Date range filtering option allows working with records between any two dates. By default, the date range filtering option is set to the current date and the date of the first record. An alternative to specific dates, users can choose preset date ranges such as "last 7 days" or "last 30 days." 2) Filtering by source simulation option displays a list of simulation models that are used as a base model by others. When the user clicks on any source simulation, the user interface then brings simulations originating from the clicked simulation. This option is specifically useful to analyze different simulations departing from the same base model (e.g., simulation exercises in CLOUDES). 3) Filtering by simulation user option lists all users with their expertise, industry, number of simulation they built, and the number of sessions they spent. Clicking on user records brings all simulation records belonging to that user. Lastly, 4) filtering by search keyword option operates on the lists generated by the last two filtering options. Matching records stay while others disappear.

Selection is considered when an analyst picks multiple specific simulation activity records that cannot be displayed altogether using current filtering options. In these cases, the analyst groups these records under a custom dataset entry. Dataset entry management is handled in a different user interface. When an analyst adds a new dataset entry to this page, the entry also appears in the filtering page allowing specific entries to be added to the dataset. Later, dataset entries can be utilized for analysis purposes. After performing 
simulation-filtering options, the console provides a list of simulation record entries. From this list, the analyst can display any record in the user interface for a quick inspection. Figure 3 depicts an example model display.

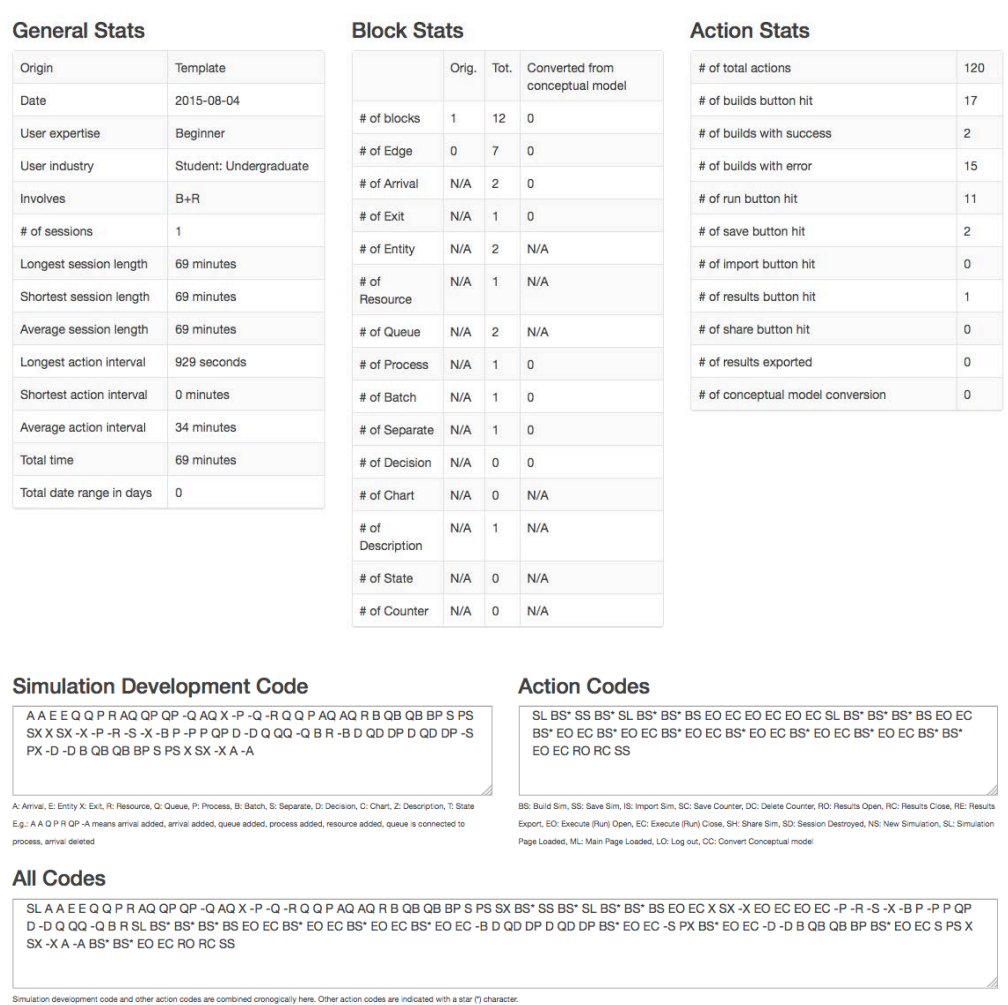

Figure 3: Simulation activity record display interface displaying an example simulation summary.

The data export capability of the console allows the user to utilize the simulation activity data in other tools. With this being the purpose, the console provides export capabilities for different cases. Dataset interface, for instance, can export simulation activity data grouped under datasets. Download bulk data screen (Figure 4 ), on the other hand, serves two types of data export capabilities with minimal filtering options. The first capability allows one to export simulation activity data, optionally filtered by the user industry, expertise, and country. We specifically use this option, for instance, when we want first to inspect all the data and filter it using another tool. The second capability is to export different data than simulation activity. This data is called sequence data, which holds aggregate sequential patterns that users follow when navigating the website. Each distinct pattern is displayed, and the number of times that the patterns are shown. All these data are in tabular format when the user downloads it.

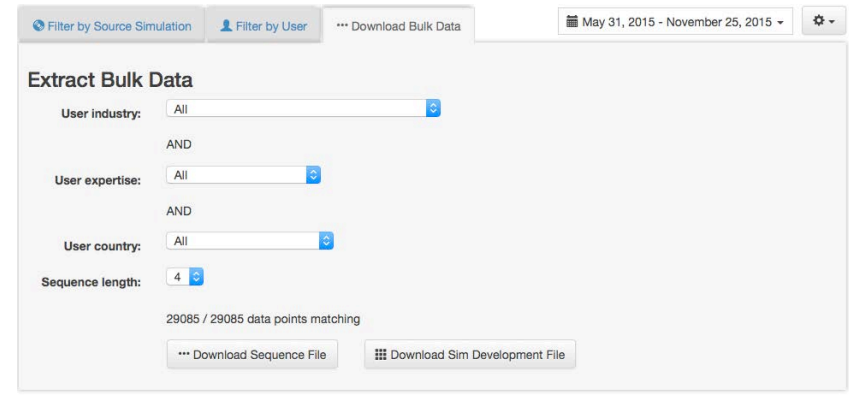

Figure 4: Simulation activity record display interface. 


\section{PRELIMINARY RESULTS}

Considering the fields provided in Table 3, we explore whether any of those fields or metrics (generated using those fields) differentiate between simulations developed by people with different expertise levels. This information is critical because it can allow us to auto-detect expertise level and customize the tool accordingly, among other potential uses. Based on our simulation development and teaching expertise, we hypothesize that the fields and metrics in Table 4 may serve as differentiating factors for simulations developed by different expertise groups. We note that normalized factors are added to make sure the scale of variable values does not affect the results.

Table 4: Factors to be explored. We note that these factors are all calculated per simulation.

\begin{tabular}{|c|c|}
\hline Factor Category & Factor list \\
\hline Temporal factors & $\begin{array}{ll}\text { - } & \text { Total time spent } \\
\text { - } & \text { Total days past } \\
\text { - } & \text { Average inter-event time } \\
\text { - } & \text { Number of sessions } \\
\text { - } & \text { Average session time }\end{array}$ \\
\hline $\begin{array}{l}\text { Block/edge } \\
\text { addition/deletion events }\end{array}$ & $\begin{array}{l}\text { - } \quad \text { Block addition per minute } \\
\text { - Number of block addition event } \\
\text { - Number of edge addition event } \\
\text { - Number of block deletion event }\end{array}$ \\
\hline Number of blocks/edges & $\begin{array}{ll}\text { - } & \text { Number of total blocks } \\
\text { - } & \text { Number of edges } \\
\text { - } & \text { Number of advanced (i.e., separate, batch, and decision) blocks } \\
\text { - } & \text { Number of batch block } \\
\text { - } & \text { Number of separate block } \\
\text { - } & \text { Number of decision block }\end{array}$ \\
\hline Other factors & $\begin{array}{ll}\text { - } & \text { Number of event } \\
\text { - } & \text { Number of build error } \\
\text { - } & \text { Number of build success } \\
\text { - } & \text { Number of simulation run attempts }\end{array}$ \\
\hline Normalized factors & $\begin{array}{ll}\text { - } & \text { Percentage of block addition events } \\
\text { - } & \text { Percentage of edge addition events } \\
\text { - } & \text { Percentage of block deletion events } \\
\text { - } & \text { Percentage of advanced blocks } \\
\text { - } & \text { Percentage of successful builds } \\
\text { - } & \text { Percentage of unsuccessful builds } \\
\end{array}$ \\
\hline
\end{tabular}

We run the one-way analysis of variance (ANOVA) tests on simulations developed by users with different expertise groups. We first investigate simulations developed by beginners $(N=814)$ vs. simulations developed by intermediate users $(N=220)$. The results gathered from the analysis tests the null hypothesis of the indifference in the mentioned factors between simulations built by the two groups. We also conduct 
the same analysis for advanced $(N=36)$ and expert $(N=30)$ groups. Expert users are assumed to have superior simulation development knowledge compared to the other three groups.

Based on the ANOVA results, we found four factors with statistically significant $(p \leq .05)$ difference for simulations built by beginners vs. intermediates. Both the number of build error and the percentage of build error are higher for simulations built by beginner users. On average, beginner users have $\approx 30 \%$ more errors when they try to build (i.e., execute) their simulation models. From an educational standpoint, a simulation course instructor can track simulation build error percentages over time and take that into account when deciding whether the student becomes more proficient. Other factors we found are the percentage of block addition and the percentage of edge addition events. Both factors show that beginner users use block and edge addition events $\approx 40 \%$ more than intermediate users on average. This particular result suggests that a simulation learner adds fewer blocks to the model while becoming more proficient. This result is intuitive as this fact can be associated with the situation that beginner programmers tend to write longer codes even for relatively easy tasks. We want to emphasize that these results compare users with their very beginning of simulation learning experience, and users have some simulation development knowledge both declared when they register to CLOUDES.

The second set of ANOVA results we gathered are from simulations that are built by advanced vs. expert users (see supplemental). We identify six factors differentiating these two groups. The majority of these factors are based on time spent on the system and the usage of the tool. For instance, on average, advanced users make approximately three times more visits to CLOUDES based on the number of sessions. Further, the total time advanced users spent is approximately four times longer than expert users. Finally, the number of events that are generated by advanced users is $\approx 5.5$ times more than expert users. These results suggest that expert users do not embrace CLOUDES. In fact, by design, CLOUDES mainly targets inexperienced simulation learners to teach them simulation concepts (Padilla et al. 2014) and have a limited number of blocks and advanced features. Therefore, we believe that the results we gather with this analysis are an artifact of the lack of use by experts.

These results give us some insights that such modeler data can be useful to uncover differences between expertise groups. While these results are intuitive, we want to be cautious because simulation models developed by these users do not necessarily represent the same system. A more controlled environment with users developing the same model would give us a better comparison point. We are planning to design such studies in the future.

\section{FUTURE DIRECTIONS AND LESSONS LEARNED}

In this paper, we showed one possible way of using the modeler data to compare between different expertise levels, while there are other ways to elicit this data. We identified four other broader uses of such modeler data to give modeling and simulation community some future directions.

- Learning simulation development styles: It is a fact that software developers show specific development patterns, making it possible to attribute authorship (Chen et al. 2010). Given that simulation creation on CLOUDES is more restricted compared to free-style coding in generalpurpose languages, it is possible to identify and classify different simulation development styles. Sequence mining of actions has the potential to reveal such information (Gabadinho et al. 2009). If development styles can be attributed to user expertise, it would be potentially possible to classify a user's expertise without asking them in the first place. We are planning to investigate such information in a future study.

- Measuring modelers' learning progression: A modeler can be classified based on development styles as described above or based on self-reported expertise levels. Development sequence data or simulation action data can be used to train a classifier, where the class would be the expertise level. It should be potentially possible to detect when a user's simulation development data looks more 
similar to users with greater expertise levels. The user's latest data can be used to measure the progression, instead of the entire set.

- User engagement and gamification: In addition to our initial insights reported, a number of failed simulation building actions may suggest a trial-and-error approach against traditional established processes. In this respect, simulation tools and educational exercises may consider ways to improve user engagement. Especially, a data-driven user engagement approach can facilitate learning patterns of success automatically from the collected modeler data and suggest new users options to keep them engaged. Gamification is one way to establish such engagement as our previous efforts support (Padilla et al. 2016, Padilla et al. 2017). Simulation tools like CLOUDES can offer gamelike engagement mechanisms in which users collect digital badges by creating certain types of models, running a model with different parameter settings, or running replications and displaying the results.

- Improving the tool: One of the challenges we faced when developing CLOUDES was to make the user interface as user-friendly as possible. This is a challenging task because simulations require a large number of settings to be provided. We can use basic statistics of the data to improve the user interface by identifying commonly used blocks, rarely used blocks, and common sequential user actions. For instance, commonly used blocks can be emphasized more, while rarely used blocks can be made hidden initially. For the sequential user actions, the user interface may ask the modeler if they want to do a commonly seen action. For instance, if users mostly add resources after a process, the tool can make this consecutive actions more user-friendly.

While having many potential benefits, conducting this research was quite challenging. Here we provide some lessons we learned while implementing this modeler data collection system.

First and foremost, identifying what data to capture is a challenging task as we are not able to locate studies from the simulation community tackle similar problems. We initially captured a limited number of actions and related data but later realized that we were missing some crucial aspects, such as capturing the up-todate model state when a user makes a certain action. Moreover, since CLOUDES is web-based, we sometimes had nightly updates and introduced new features, these additions altered the data we capture from users. Keeping track of the changing data fields makes it very challenging to handle corner cases. We recommend the community to conduct a pilot process and to make sure that the collected data is easily usable by analysis tools.

Second, we observed unexpected user behavior. For instance, we assumed that a user would only use one browser tab for a particular model. To our surprise, we observed anomalies in our data, which contradicts this assumption. With a closer inspection, we observed that some users open the model in multiple tabs at the same time, and even some users open multiple browsers at the same time. These observations made us add the "PageId" column, which partly solved the challenge. The complete solution involves either not allowing multiple tabs/browser windows to be open at the same time or implementing a synchronization mechanism between different pages. Notably, the latter is a lengthy process.

Finally, the increase in collected data size also increased the processing time for the user behavior console. Since we have a data column that may have different information in JSON format, we had to develop a parser that behaves differently for different types of actions. While this provides certain flexibility, it becomes computationally heavy to handle a large number of lines of record. For instance, when our data size passed 150,000 records, the parsing system we described in section 3.2 became comparatively slow. To address this issue, we need to export data into multiple SQL tables, which is quite challenging to handle, given that we changed data format frequently. An alternative to that would be using a no-SQL database system (Chodorow 2013), which is suitable to deal with such data. 
Kavak, Padilla, Diallo, and Barraco

\section{APPENDIX}

ANOVA results are available online as a supplementary material to this paper. Download address:

http://hamdikavak.com/download/modeling the modeler_supplemental.pdf

\section{REFERENCES}

Cant, Robyn P., and Simon J. Cooper. 2010. "Simulation-based learning in nurse education: systematic review." Journal of Advanced Nursing 66 (1):3-15. doi: 10.1111/j.1365-2648.2009.05240.x.

Chen, R., L. Hong, C. Chunyan Lü, and W. Deng. 2010. "Author Identification of Software Source Code with Program Dependence Graphs." 2010 IEEE 34th Annual Computer Software and Applications Conference Workshops, 19-23 July 2010.

Chodorow, K. 2013. MongoDB: The Definitive Guide: Powerful and Scalable Data Storage: O'Reilly Media.

Clauset, Aaron, Cosma Rohilla Shalizi, and M. E. J. Newman. 2009. "Power-Law Distributions in Empirical Data." SIAM Review 51 (4):661-703. doi: 10.1137/070710111.

Csikszentmihalyi, Mihaly, Sami Abuhamdeh, and Jeanne Nakamura. 2014. "Flow." In Flow and the Foundations of Positive Psychology: The Collected Works of Mihaly Csikszentmihalyi, edited by Mihaly Csikszentmihalyi, 227-238. Dordrecht: Springer Netherlands.

Deuro, Justin, Christopher J. Lynch, Hamdi Kavak, and Jose J. Padilla. 2017. "Incorporating Sound in Simulations." Winter Simulation Conference, Las Vegas, NV, USA, 2017.

Fields, Deborah A., Lisa Quirke, Janell Amely, and Jason Maughan. 2016. Combining Big Data and Thick Data Analyses for Understanding Youth Learning Trajectories in a Summer Coding Camp, Proceedings of the 47th ACM Technical Symposium on Computing Science Education. Memphis, Tennessee, USA: Association for Computing Machinery.

Gabadinho, Alexis, Gilbert Ritschard, Matthias Studer, and Nicolas S Müller. 2009. "Mining sequence data in R with the TraMineR package: A user's guide." Geneva: Department of Econometrics and Laboratory of Demography, University of Geneva.

Padilla, J. J., S. Y. Diallo, and H. M. Garcia. 2018. "From Analogue to Digital: Creating Simulations Through Conceptualization Boards." 2018 Winter Simulation Conference (WSC), 9-12 Dec. 2018.

Padilla, Jose J., Saikou Y. Diallo, Hamdi Kavak, Anthony Barraco, Christopher J. Lynch, and Hamdi Kavak. 2014. "Cloud-based simulators: Making simulations accessible to non-experts and experts alike." 2015.

Padilla, Jose J., Christopher J. Lynch, Hamdi Kavak, Saikou Y. Diallo, Ross J. Gore, Anthony Barraco, and Bakari Jenkins. 2016. "Using Simulation Games for Teaching and Learning Discrete-Event Simulation." Winter Simulation Conference, Arlington, VA, USA, 2016.

Padilla, Jose J., Christopher J. Lynch, Hamdi Kavak, Shawn Evett, Devon Nelson, Calvin Carson, and Joshua del Villar. 2017. "Storytelling and Simulation Creation." Winter Simulation Conferene, Las Vegas, NV, USA, 2017.

Repenning, Alexander, David C. Webb, Kyu Han Koh, Hilarie Nickerson, Susan B. Miller, Catharine Brand, Ian Her Many Horses, Ashok Basawapatna, Fred Gluck, Ryan Grover ., and et al. 2015. Scalable Game Design: A Strategy to Bring Systemic Computer Science Education to Schools through Game Design and Simulation Creation. Vol. 15: Association for Computing Machinery. 
Wieman, Carl E., Wendy K. Adams, and Katherine K. Perkins. 2008. "PhET: simulations that enhance learning." Science 322 (5902):682-683. doi: 10.1126/science.1161948.

\section{AUTHOR BIOGRAPHIES}

HAMDI KAVAK is an Assistant Professor at the Department of Computational and Data Sciences at George Mason University (GMU) and a member of the Center for Social Complexity at GMU. His research focuses on designing novel data-driven computational models to tackle domain-specific problems in cybersecurity and urban systems. He holds Ph.D. and M.E. degrees in M\&S from Old Dominion University. His email address is hkavak@gmu.edu and his website is http://www.hamdikavak.com.

JOSE J PADILLA is Research Associate Professor at the Virginia Modeling, Analysis and Simulation Center at Old Dominion University. His research focuses 1) on the combination of methods and technological innovations to make simulations accessible to non-simulationists and 2) on the application of those methods to study areas like cybersecurity and forced migration. His email address is jpadilla@odu.edu.

SAIKOU Y. DIALLO is a Research Associate Professor at the Virginia Modeling, Analysis, and Simulation Center (VMASC) where he serves as the head of the Simulated Empathy lab. He is also an adjunct Professor of Modeling, Simulation, and Visualization Engineering at Old Dominion University (ODU). He received his $\mathrm{MS}$ and $\mathrm{PhD}$ in Modeling and Simulation from ODU. His email address is sdiallo@odu.edu and his web page is http://www.odu.edu/vmasc/research/simulated-empathy.html.

ANTHONY M BARRACO is a Senior Project Scientist the Virginia Modeling, Analysis and Simulation Center at Old Dominion University. He received his B.S. in Computer Science from the University of Central Florida. His email is abarraco@odu.edu. 